\title{
SINGULARITIES OF ONE-PARAMETER PEDAL UNFOLDINGS OF SPHERICAL PEDAL CURVES
}

\author{
T. NISHIMURA
}

\begin{abstract}
In this paper, we present the concept of one-parameter pedal unfoldings of a pedal curve in the unit sphere $S^{2}$, and we classify their generic singularities with respect to $\mathcal{A}$-equivalence.
\end{abstract}

\section{INTRODUCTION}

Let $I$ be an open interval containing zero, and let $S^{2}$ be the unit sphere in Euclidean space $\mathbb{R}^{3}$. A $C^{\infty}$ map $\mathbf{r}: I \rightarrow S^{2}$ is called a spherical unit speed curve if $\left\|\frac{d \mathbf{r}}{d s}(s)\right\|$ is 1 for any $s \in I$. For a given spherical unit speed curve $\mathbf{r}: I \rightarrow S^{2}$, we put

$$
\mathbf{t}(s)=\frac{d \mathbf{r}}{d s}(s), \mathbf{n}(s)=\mathbf{r}(s) \times \mathbf{t}(s),
$$

where $\mathbf{r}(s) \times \mathbf{t}(s)$ denotes the vector product of $\mathbf{r}(s)$ and $\mathbf{t}(s)$. The construction clearly shows that the vector $\mathbf{t}(s)$ is perpendicular to the vector $\mathbf{r}(s)$ and that the vector $\mathbf{n}(s)$ is perpendicular to both $\mathbf{r}(s)$ and $\mathbf{t}(s)$. The map $\mathbf{n}: I \rightarrow S^{2}$ is called the spherical dual of $\mathbf{r}$; the singularities of spherical dual curves are Legendrian singularities that are relatively well investigated [1, 2, 3, 4, 5, 21.

For a point $P \in S^{2}$, let $E_{P}$ denote the set $\left\{X \in S^{2} \mid P \cdot X=0\right\}$, where $P \cdot X$ denotes the scalar product of $P$ and $X$. For a given spherical unit speed curve $\mathbf{r}: I \rightarrow S^{2}$, consider a point $P$ of $S^{2}-\{ \pm \mathbf{n}(s) \mid s \in I\}$, where $\mathbf{n}$ is the spherical dual of $\mathbf{r}$. The spherical pedal curve relative to the point $P$ for a given spherical unit speed curve $\mathbf{r}: I \rightarrow S^{2}$ is a curve obtained by mapping $s \in I$ to the nearest point in $E_{\mathbf{n}(s)}$ from $P$. The pedal curve relative to $P$ for $\mathbf{r}$ is denoted by ped $_{\mathbf{r}, P}$, and the point $P$ is called the pedal point of the pedal curve $\operatorname{ped}_{\mathbf{r}, P}$. Note that all points in $E_{\mathbf{n}(s)}$ are equidistant from $\pm \mathbf{n}(s)$; hence, the point $P$ must lie outside $\{ \pm \mathbf{n}(s) \mid s \in I\}$ to satisfy the definition of $\operatorname{ped}_{\mathbf{r}, P}$. The classification of singularities of spherical pedal curves can be found in literature [17, 18, 19.

Suppose that the location of the pedal point $P$ moves smoothly, depending on one-parameter $\lambda \in J$, where $J$ is an open interval containing zero in $\mathbb{R}$. In other words, suppose that there exist an open interval $J$ containing zero and a $C^{\infty}$ immersion $P: J \rightarrow S^{2}$. Then, the pedal unfolding of the pedal curve ped $d_{\mathbf{r}, P(0)}$ can be defined as the map $U n-p e d_{\mathbf{r}, P}: I \times J \rightarrow S^{2} \times J$, given by

$$
U n-\operatorname{ped}_{\mathbf{r}, P}(s, \lambda)=\left(\operatorname{ped}_{\mathbf{r}, P(\lambda)}(s), \lambda\right) .
$$

Two $C^{\infty}$ map-germs $f, g:\left(\mathbb{R}^{n}, 0\right) \rightarrow\left(\mathbb{R}^{p}, 0\right)$ are said to be $\mathcal{A}$-equivalent if there exist germs of $C^{\infty}$-diffeomorphisms $h_{1}:\left(\mathbb{R}^{n}, 0\right) \rightarrow\left(\mathbb{R}^{n}, 0\right)$ and $h_{2}:\left(\mathbb{R}^{p}, 0\right) \rightarrow\left(\mathbb{R}^{p}, 0\right)$ such that $f \circ h_{1}=h_{2} \circ g$. For a spherical unit speed curve germ $\mathbf{r}:(I, 0) \rightarrow S^{2}$, we put $\kappa(s)=\mathbf{n}(s) \cdot \mathbf{t}^{\prime}(s)$, where $\mathbf{t}^{\prime}$ denotes

2010 Mathematics Subject Classification. 58K40, 58K50.

Key words and phrases. spherical pedal curve, pedal unfolding, cross-cap, $S_{k}^{ \pm}$singularity, Chen-MatumotoMond singularity, cuspidal edge, cuspidal cross-cap, cuspidal $S_{k}^{ \pm}$singularity, cuspidal Chen-Matumoto-Mond singularity. 
TABLe 1. Normal forms of $\mathcal{A}$-simple monogerms $\left(\mathbb{R}^{2}, 0\right) \rightarrow\left(\mathbb{R}^{3}, 0\right)([15])$

\begin{tabular}{lc}
\hline Germ & Name \\
\hline$f(s, \lambda)=\left(s, s^{2}, \lambda\right)$ & Immersion \\
$f(s, \lambda)=\left(s^{3}+\lambda s, s^{2}, \lambda\right)$ & Cross-cap $\left(S_{0}\right)$ \\
$f(s, \lambda)=\left(s^{3} \pm \lambda^{k+1} s, s^{2}, \lambda\right),(k \geq 1)$ & $S_{k}^{ \pm}$ \\
$f(s, \lambda)=\left(\lambda^{2} s \pm s^{2 k+1}, s^{2}, \lambda\right),(k \geq 2)$ & $B_{k}^{ \pm}$ \\
$f(s, \lambda)=\left(\lambda s^{3} \pm \lambda^{k} s, s^{2}, \lambda\right),(k \geq 3)$ & $C_{k}^{ \pm}$ \\
$f(s, \lambda)=\left(\lambda^{3} s+s^{5}, s^{2}, \lambda\right)$ & $F_{4}$ \\
$f(s, \lambda)=\left(\lambda s+s^{3 k-1}, s^{3}, \lambda\right),(k \geq 2)$ & $H_{k}$ \\
\hline
\end{tabular}

the derivative of $\mathbf{t}$. Then, the point $\mathbf{r}(0)$ is called the inflection point (resp., ordinary inflection point) if $\kappa(0)=0$ holds (resp., $\kappa(0)=0$ and $\kappa^{\prime}(0) \neq 0$ hold). For any $k \geq 0$, a $C^{\infty}$ immersed curve germ $P:(J, 0) \rightarrow S^{2}$ is said to have $(k+1)$-point contact with $\mathbf{r}:(I, 0) \rightarrow S^{2}$ at $P(0)=\mathbf{r}(0)$ if $P(0)=\mathbf{r}(0), F \circ P(0)=(F \circ P)^{\prime}(0)=\cdots=(F \circ P)^{(k)}(0)=0$, and $(F \circ P)^{(k+1)}(0) \neq 0$ hold for any neighbourhood $U$ of $\mathbf{r}(0)$ and any non-singular $C^{\infty}$ function $F: U \rightarrow \mathbb{R}$ such that $F \circ \mathbf{r}(s)=0$ (for details on $(k+1)$ )-point contact, see [5]). It can be clearly seen that a $C^{\infty}$ immersed curve germ $P:(J, 0) \rightarrow S^{2}$ has 1 -point contact with $\mathbf{r}:(I, 0) \rightarrow S^{2}$ at $P(0)=\mathbf{r}(0)$ if and only if $P$ and $\mathbf{r}$ are transverse at $P(0)=\mathbf{r}(0)$.

Theorem 1. Let $I, J$ be open intervals containing $0 \in \mathbb{R}$, and let $\mathbf{r}: I \rightarrow S^{2}$ be a spherical unit speed curve such that $\mathbf{r}(0)$ is not an inflection point. Furthermore, let $P: J \rightarrow S^{2}$ be a $C^{\infty}$ immersion. Then, the following hold:

(1) The germ of pedal unfolding Un-ped $d_{\mathbf{r}, P}:(I \times J,(0,0)) \rightarrow S^{2} \times J$ is immersive if and only if $P(0) \neq \mathbf{r}(0)$.

(2) The germ of pedal unfolding Un-ped $d_{\mathbf{r}, P}:(I \times J,(0,0)) \rightarrow S^{2} \times J$ is $\mathcal{A}$-equivalent to the cross-cap in Table 1 if und only if $P(0)=\mathbf{r}(0)$ and $P, \mathbf{r}$ are transverse at $P(0)=\mathbf{r}(0)$.

(3) The germ of pedal unfolding Un-ped $d_{\mathbf{r}, P}:(I \times J,(0,0)) \rightarrow S^{2} \times J$ is $\mathcal{A}$-equivalent to $S_{k}^{ \pm}$ in Table 1 if and only if $P(0)=\mathbf{r}(0)$ and $P$ has $(k+1)$-point contact with $\mathbf{r}$ at $0 \in J$ $(k \geq 1)$.

(4) The $\mathcal{A}$-equivalence classes of map-germs $B_{k}^{ \pm}, C_{k}^{ \pm}, F_{4}$, and $H_{k}$ in Table 1 can never be realized as singularities of the pedal unfolding $U n-p e d_{\mathbf{r}, P}$.

(5) The germ of pedal unfolding Un-ped $\mathrm{r}_{\mathbf{r}, P}:(I \times J,(0,0)) \rightarrow S^{2} \times J$ is $\mathcal{A}$-equivalent to the cuspidal edge in Table 2 if and only if $P(0)=\mathbf{r}(0)$ and $(P(J), P(0))$ coincides with $(\mathbf{r}(I), \mathbf{r}(0))$ as set-germs.

If $k$ is even, then it can be clearly seen that $S_{k}^{+}$is $\mathcal{A}$-equivalent to $S_{k}^{-}$[15]. On the other hand, $S_{k}^{+}$is not $\mathcal{A}$-equivalent to $S_{k}^{-}$if $k$ is odd. Figure 2 shows that the curvature of $\mathbf{r}$ at zero is greater than the curvature of $P$ at zero if and only if the pedal unfolding $U n-p e d_{\mathbf{r}, P}$ is $\mathcal{A}$-equivalent to $S_{k}^{-}$. Since $S_{1}^{ \pm}$has been investigated independently in [6], it is reasonable to classify the $\mathcal{A}$-equivalence class of $S_{1}^{ \pm}$as Chen-Matumoto-Mond singularity.

Theorem 2. Let $I, J$ be open intervals containing $0 \in \mathbb{R}$, and let $\mathbf{r}: I \rightarrow S^{2}$ be a spherical unit speed curve such that $\mathbf{r}(0)$ is an ordinary inflection point. Furthermore, let $P: J \rightarrow S^{2}$ be a $C^{\infty}$ immersion. Then, the following hold:

(1) The germ of pedal unfolding Un-ped $\mathbf{r}_{\mathbf{r}, P}:(I \times J,(0,0)) \rightarrow S^{2} \times J$ is $\mathcal{A}$-equivalent to the cuspidal edge in Table 2 if and only if $P(0) \notin E_{\mathbf{n}(0)}$. 

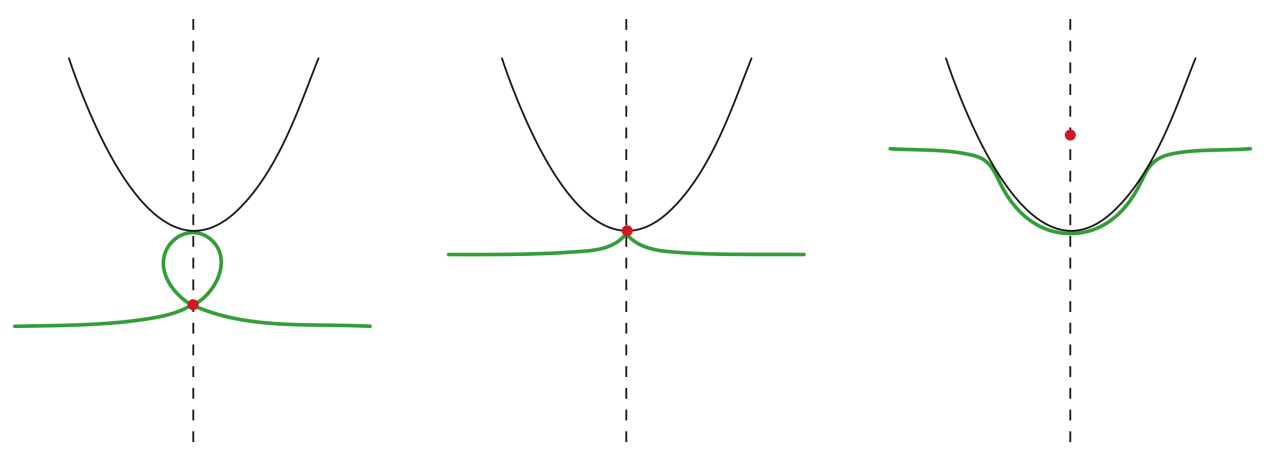

Figure 1. Cross-cap. Left: $\lambda=-\varepsilon$, Center: $\lambda=0$, Right: $\lambda=\varepsilon$.
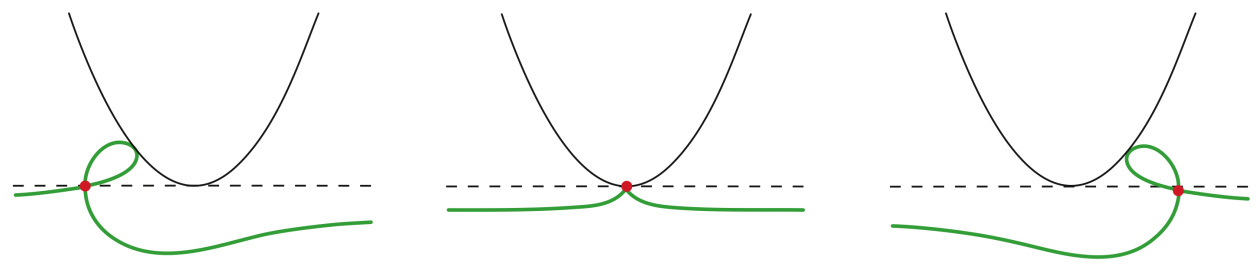

FIgURE 2. $S_{1}^{-}$. Left: $\lambda=-\varepsilon$, Center: $\lambda=0$, Right: $\lambda=\varepsilon$.

TABLE 2 .

\begin{tabular}{lc}
\hline Germ & Name \\
\hline$g(s, \lambda)=\left(s^{3}, s^{2}, \lambda\right)$ & Cuspidal edge \\
$g_{0}^{+}(s, \lambda)=\left(s^{5}+\lambda s^{3}, s^{2}, \lambda\right)$ & Cuspidal cross-cap (Cuspidal $\left.S_{0}\right)$ \\
$g_{k}^{ \pm}(s, \lambda)=\left(s^{5} \pm \lambda^{k+1} s^{3}, s^{2}, \lambda\right),(k \geq 1)$ & Cuspidal $S_{k}^{ \pm}$ \\
\hline
\end{tabular}

(2) The germ of pedal unfolding Un-ped $d_{\mathbf{r}, P}:(I \times J,(0,0)) \rightarrow S^{2} \times J$ is $\mathcal{A}$-equivalent to the cuspidal cross-cap in Table 2 if und only if $P(0) \in E_{\mathbf{n}(0)}-\{\mathbf{r}(0)\}$ and $P$ is transverse to $E_{\mathbf{n}(0)}$ at $P(0)$.

(3) The germ of pedal unfolding Un-ped $\mathbf{r}_{\mathbf{r}, P}:(I \times J,(0,0)) \rightarrow S^{2} \times J$ is $\mathcal{A}$-equivalent to cuspidal $S_{k}^{ \pm}(k \geq 1)$ in Table 2 if and only if $P(0) \in E_{\mathbf{n}(0)}-\{\mathbf{r}(0)\}$ and $P$ has $(k+1)$ point contact with $E_{\mathbf{n}(0)}(k \geq 1)$.

As in the case of $S_{k}^{ \pm}$singularities, it can be clearly seen that cuspidal $S_{k}^{+}$singularity is $\mathcal{A}$ equivalent to cuspidal $S_{k}^{-}$singularity if $k$ is even. On the other hand, cuspidal $S_{k}^{+}$singularity is not $\mathcal{A}$-equivalent to cuspidal $S_{k}^{-}$singularity if $k$ is odd. Figure 4 shows that for a sufficiently small positive real number $\varepsilon$, there exists a positive real number $\delta$ such that the union of tangent lines $\cup_{s \in(-\varepsilon, \varepsilon)} E_{\mathbf{n}(s)}$ contains the images $P((-\delta, \delta))$ if and only if the map-germ Un-ped $d_{\mathbf{r}, P}$ : $(I \times J,(0,0)) \rightarrow S^{2} \times J$ is $\mathcal{A}$-equivalent to cuspidal $S_{k}^{-}$singularity. Since map-germ $g_{0}^{+}$singularity 

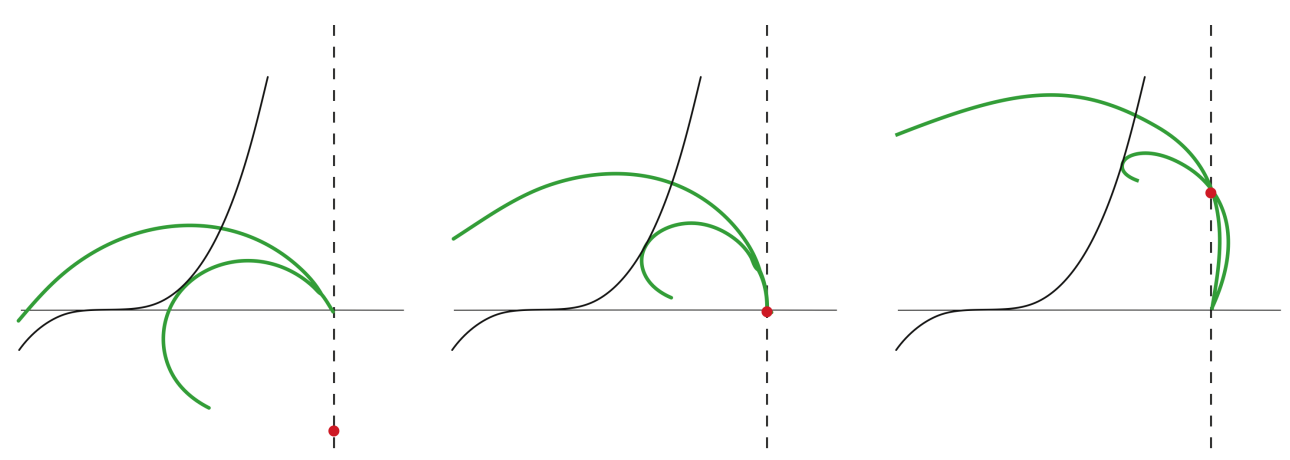

Figure 3. Cuspidal cross-cap. Left: $\lambda=-\varepsilon$, Center: $\lambda=0$, Right: $\lambda=\varepsilon$.
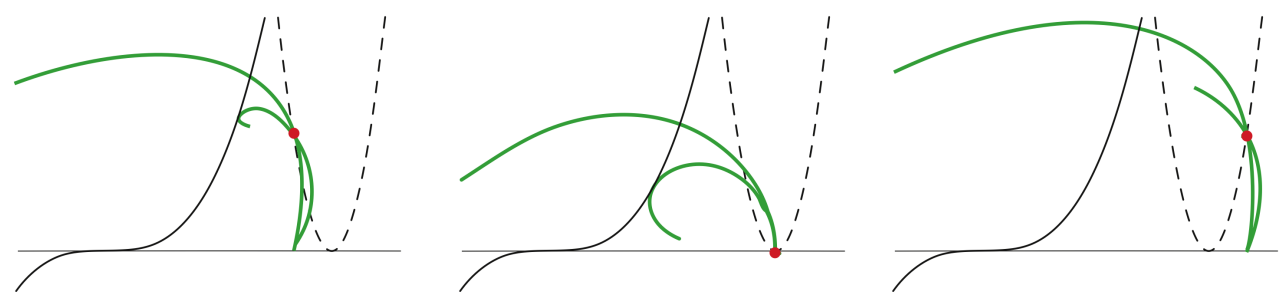

Figure 4. Cuspidal $S_{1}^{-}$. Left: $\lambda=-\varepsilon$, Center: $\lambda=0$, Right: $\lambda=\varepsilon$.

is known as the normal form of the cuspidal cross-cap (see 11]), it is reasonable to classify the $\mathcal{A}$-equivalence class of the map-germ $g_{k, \pm}$ (resp., $g_{1, \pm}$ ) as cuspidal $S_{k}^{ \pm}$singularity (resp., cuspidal Chen-Matumoto-Mond singularity).

It can be clearly seen that the cuspidal edge, cuspidal cross-cap, and cuspidal $S_{k}^{ \pm}$are not finitely $\mathcal{A}$-determined (but finitely $\mathcal{K}$-determined) by the Mather-Gaffney geometric characterization of finite determinacy, even though $S_{k}^{ \pm}$singularity is $(k+2)$ - $\mathcal{A}$-determined [15] (for the definition of finite determinacy and Mather-Gaffney geometric characterization, see [23]). Thus, in order to prove Theorems 1 and 2 in a unified manner, it is difficult to directly use the standard techniques of the finite determinacy theory developed in [8, 9, 10, 13, 14, 15, 20, 23].

On the other hand, Saji succeeded in obtaining simple criteria for Chen-Matumoto-Mond singularity and cuspidal $S_{k}^{ \pm}$-singularities [22]. Although Saji's criteria are useful, the criteria for $S_{k}^{ \pm}$singularities $(k \geq 2)$ have not been provided by him; therefore, Saji's criteria are not suited to our purpose. In this study, we plan to develop a unified method for proving Theorems 1 and 2 ; hence, we adopt a recognition criterion for map-germs that appear as singularities of pedal unfoldings. It is important to note that this criterion has already been presented in a suitable form in [15].

The preliminary work required to prove Theorems 1 and 2 is presented in Section 2. Theorems 1 and 2 are proved in Sections 3 and 4, respectively. 
The author would like to extend his gratitude to Y. Sakemi for providing useful graphics.

\section{Preliminaries}

2.1. Spherical pedal curves. Let $I, S^{2}$, and $\mathbf{r}: I \rightarrow S^{2}$ be an interval containing zero, the unit sphere in $\mathbb{R}^{3}$, and a spherical unit speed curve respectively. Furthermore, let $\mathbf{t}: I \rightarrow S^{2}$, $\mathbf{n}: I \rightarrow S^{2}$ be map-germs, as described in Section 1. Then, we have the following Serret-Frenet type formula.

Lemma 2.1 ([17]).

$$
\left(\begin{array}{c}
\mathbf{r}^{\prime}(s) \\
\mathbf{t}^{\prime}(s) \\
\mathbf{n}^{\prime}(s)
\end{array}\right)=\left(\begin{array}{ccc}
0 & 1 & 0 \\
-1 & 0 & \kappa(s) \\
0 & -\kappa(s) & 0
\end{array}\right)\left(\begin{array}{c}
\mathbf{r}(s) \\
\mathbf{t}(s) \\
\mathbf{n}(s)
\end{array}\right)
$$

By Lemma 2.1, the dual curve germ $\mathbf{n}:(I, 0) \rightarrow S^{2}$ is non-singular at 0 if and only if $\kappa(0) \neq 0$. By using Lemma 2.1 recursively, we obtain the following:

Lemma 2.2. (1) Suppose that $\kappa(0) \neq 0$. Then, the properties $\mathbf{r}(0) \cdot \mathbf{n}^{\prime}(0)=0, \mathbf{r}(0) \cdot \mathbf{n}^{\prime \prime}(0) \neq$ 0 , and $\mathbf{t}(0) \cdot \mathbf{n}^{\prime}(0) \neq 0$ hold.

(2) Suppose that $\kappa(0)=0$ and $\kappa^{\prime}(0) \neq 0$. Then, the properties $\mathbf{r}(0) \cdot \mathbf{n}^{\prime}(0)=\mathbf{r}(0) \cdot \mathbf{n}^{\prime \prime}(0)=0$, $\mathbf{r}(0) \cdot \mathbf{n}^{(3)}(0) \neq 0, \mathbf{t}(0) \cdot \mathbf{n}^{\prime}(0)=0$, and $\mathbf{t}(0) \cdot \mathbf{n}^{\prime \prime}(0) \neq 0$ hold .

Let $P$ be a point of $S^{2}-\{ \pm \mathbf{n}(s) \mid s \in I\}$.

Lemma 2.3 ([17]). The pedal curve of $\mathbf{r}$ relative to the pedal point $P$ is given by the following expression:

$$
\operatorname{ped}_{\mathbf{r}, P}(s)=\frac{1}{\sqrt{1-(P \cdot \mathbf{n}(s))^{2}}}(P-(P \cdot \mathbf{n}(s)) \mathbf{n}(s)) .
$$

Let $\Psi_{P}$ be the $C^{\infty}$ map from $S^{2}-\{ \pm P\}$ to $S^{2}$, given by

$$
\Psi_{P}(X)=\frac{1}{\sqrt{1-(P \cdot X)^{2}}}(P-(P \cdot X) X)
$$

The map $\Psi_{P}$, which has been introduced and used in [17, 18, 19] (the hyperbolic version of $\Psi_{P}$ has been introduced and investigated independently in [12]), has the following distinctive properties :

(1) $X \cdot \Psi_{P}(X)=0$ for any $X \in S^{2}-\{ \pm P\}$.

(2) $\Psi_{P}(X) \in \mathbb{R} P+\mathbb{R} X$ for any $X \in S^{2}-\{ \pm P\}$.

(3) $P \cdot \Psi_{P}(X)>0$ for any $X \in S^{2}-\{ \pm P\}$.

By property $3, \Psi_{P}\left(S^{2}-\{ \pm P\}\right)$ lies inside the open hemisphere centered at $P$. By properties 1 and $2, \Psi_{P}\left(E_{P}\right)=P$. Let the open hemisphere centered at $P$ be denoted by $H_{P}$, and put $B_{P}=$ $\pi\left(S^{2}-\{ \pm P\}\right)$, where $\pi: S^{2} \rightarrow P^{2}(\mathbb{R})$ is the canonical projection. Since $\Psi_{P}(X)=\Psi_{P}(-X)$, the map $\Psi_{P}$ canonically induces the map $\widetilde{\Psi}_{P}: B_{P} \rightarrow H_{P}$. Then, by Lemma 2.3 , ped,$P$ is factored into three maps as follows:

$$
\operatorname{ped}_{\mathbf{r}, P}(s)=\widetilde{\Psi}_{P} \circ \pi \circ \mathbf{n}(s) .
$$

Let $p: B \rightarrow \mathbb{R}^{2}$ be the blow up centered at the origin in $\mathbb{R}^{2}$.

Lemma 2.4 ([17]). Let $P$ be a point of $S^{2}$. Then, there exist $C^{\infty}$ diffeomorphisms $h_{1}: B_{P} \rightarrow B$ and $h_{2}: H_{P} \rightarrow \mathbb{R}^{2}$ such that the equality $h_{2} \circ \widetilde{\Psi}_{P}=p \circ h_{1}$ holds, and the set $\pi\left(E_{P}\right)$ is mapped to the exceptional set of $p$ by $h_{1}$. 
2.2. Criterion for recognition problem due to Mond. Let $T: \mathbb{R}^{2} \rightarrow \mathbb{R}^{2}$ be the linear transformation of the form $T(s, \lambda)=(-s, \lambda)$. Two $C^{\infty}$ function germs $p_{1}, p_{2}:\left(\mathbb{R}^{2}, 0\right) \rightarrow(\mathbb{R}, 0)$ are said to be $\mathcal{K}^{T}$-equivalent if there exist a germ of $C^{\infty}$ diffeomorphism $h:\left(\mathbb{R}^{2}, 0\right) \rightarrow\left(\mathbb{R}^{2}, 0\right)$ of the form $h \circ T=T \circ h$ and a $C^{\infty}$ function-germ $M:\left(\mathbb{R}^{2},(0,0)\right) \rightarrow \mathbb{R}$ of the form $M \circ T=M$, $M(0,0) \neq 0$ such that $p_{1} \circ h(s, \lambda)=M(s, \lambda) p_{2}(s, \lambda)([15])$.

Theorem 3 ([15]). Two $C^{\infty}$ map-germs of the following form

$$
f_{i}(s, \lambda)=\left(s p_{i}\left(s^{2}, \lambda\right), s^{2}, \lambda\right) \quad \text { where } p_{i}\left(s^{2}, \lambda\right) \notin m_{2}^{\infty}, \quad(i=1,2)
$$

are $\mathcal{A}$-equivalent if and only if the function-germs $p_{i}\left(s^{2}, \lambda\right)$ are $\mathcal{K}^{T}$-equivalent.

Note that Theorem 3 provides a criterion for the $\mathcal{A}$-equivalence of $C^{\infty}$ map-germs of the forms $(s, \lambda) \mapsto\left(\varphi(s, \lambda), s^{2}, \lambda\right)\left(\varphi:\left(\mathbb{R}^{2}, 0\right) \rightarrow(\mathbb{R}, 0)\right.$ is a $C^{\infty}$ function-germ) on the basis of the Malgrange preparation theorem (for the Malgrange preparation theorem, see [4, 23]).

\section{Proof of Theorem 1}

Since $\mathbf{r}(0)$ is not an inflection point, the dual germ $\mathbf{n}:(I, 0) \rightarrow S^{2}$ is a $C^{\infty}$ immersive germ.

Proof of assertion 1 of Theorem 1.

Suppose that $P(0)$ does not belong to $E_{\mathbf{n}(0)}$. Then, by Lemma 2.4, the restriction $\left.\Psi_{P(0)}\right|_{S^{2}-\{ \pm P(0)\}-E_{P(0)}}$ is $C^{\infty}$ immersive. Thus, by Lemma 2.3, the map-germ $\operatorname{ped}_{\mathbf{r}, P(0)}:(I, 0) \rightarrow S^{2}$ is also $C^{\infty}$ immersive. Therefore, the map-germ $U n$-ped $d_{\mathbf{r}, P}:(I \times J,(0,0)) \rightarrow S^{2} \times J$ is also $C^{\infty}$ immersive. Next, suppose that $P(0) \in E_{\mathbf{n}(0)}-\mathbf{r}(0)$. Then, the image of the dual $\mathbf{n}$ and $E_{P(0)}$ intersect transeversely at $\mathbf{n}(0)$. Thus, by Lemmata 2.3 and 2.4 , the map-germ $\operatorname{ped}_{\mathbf{r}, P(0)}:(I, 0) \rightarrow S^{2}$ is $C^{\infty}$ immersive. Therefore, the map-germ $U n$-ped $d_{\mathbf{r}, P}:(I \times J,(0,0)) \rightarrow S^{2} \times J$ is also $C^{\infty}$ immersive.

Conversely, suppose that the map-germ $U n$-ped $\mathbf{r}_{\mathbf{r}, P}:(I \times J,(0,0)) \rightarrow S^{2} \times J$ is $C^{\infty}$ immersive. Then, in particular, the map-germ $\operatorname{ped}_{\mathbf{r}, P(0)}:(I, 0) \rightarrow S^{2}$ is also $C^{\infty}$ immersive. In order to conclude the proof of assertion 1 of Theorem 1 , it is sufficient to show that the assumption $P(0)=\mathbf{r}(0)$ implies a contradiction. The assumption $P(0)=\mathbf{r}(0)$ implies that the image of $\mathbf{n}$ is tangent to $E_{P(0)}$ at $\mathbf{n}(0)$. By Lemma 2.4, the map-germ $\operatorname{ped}_{\mathbf{r}, P(0)}:(I, 0) \rightarrow S^{2}$ must be singular; this is a contradiction.

Proof of assertion 5 of Theorem 1.

Suppose that both $P(0)=\mathbf{r}(0)$ and $(P(J), P(0))=(\mathbf{r}(I), \mathbf{r}(0))$ as set-germs hold. Then, for any $\lambda \in J$, ped $d_{\mathbf{r}, P(\lambda)}:(I, 0) \rightarrow S^{2}$ is $\mathcal{A}$-equivalent to the ordinary cusp $s \mapsto\left(s^{3}, s^{2}\right)$ by [17] (also, see [19]). Thus, by using the Malgrange preparation theorem and Theorem 3, the map-germ $U n$-ped $d_{\mathbf{r}, P}:(I \times J,(0,0)) \rightarrow S^{2} \times J$ is $\mathcal{A}$-equivalent to the cuspidal edge $(s, \lambda) \mapsto\left(s^{3}, s^{2}, \lambda\right)$.

Conversely, suppose that the map-germ $U n$-ped $d_{\mathbf{r}, P}:(I \times J,(0,0)) \rightarrow S^{2} \times J$ is $\mathcal{A}$-equivalent to the cuspidal edge. Then, in particular, for any sufficiently small $\lambda_{0} \in J$, there exists a sufficiently small $s_{0} \in I$ such that the map-germ $\operatorname{ped}_{\mathbf{r}, P\left(\lambda_{0}\right)}:\left(I, s_{0}\right) \rightarrow S^{2}$ is singular. Since $\mathbf{r}(0)$ is not an inflection point, by Lemma 2.4, $E_{P\left(\lambda_{0}\right)}=S^{2} \cap\left(\mathbb{R} \mathbf{t}\left(s_{0}\right)+\mathbb{R} \mathbf{n}\left(s_{0}\right)\right)$. Therefore, $P\left(\lambda_{0}\right)=\mathbf{r}\left(s_{0}\right)$.

Proof of assertions 2 and 3 of Theorem 1.

By composing an appropriate rotation without the loss of generality, it can be assumed that $\mathbf{r}(0)=(0,1,0), \mathbf{t}(0)=(0,0,1), \mathbf{n}(0)=(-1,0,0)$. For a point $Q$ of $S^{2}$, put $H(Q)=\{X \in$ $\left.S^{2} \mid Q \cdot X \geq 0\right\}$, and let $\alpha_{\mathbf{n}(0)}: H(\mathbf{n}(0))-E_{\mathbf{n}(0)} \rightarrow\{-1\} \times \mathbb{R}^{2}$ be the central projection relative to $\mathbf{n}(0)$. Then, by Lemma 2.2, the germ of composition $\alpha_{\mathbf{n}(0)} \circ \mathbf{n}$ is of the form

$$
\alpha_{\mathbf{n}(0)} \circ \mathbf{n}(s)=\left(s+\varphi_{1}(s), s^{2}+\varphi_{2}(s)\right) \quad\left(\varphi_{j}(s)=o\left(s^{j}\right)\right) .
$$


Since $\varphi_{2}(s)=o\left(s^{2}\right)$, the map-germ given by $h\left(s \sqrt{1+\frac{\varphi_{2}(s)}{s^{2}}}\right)=s$ is a well-defined germ of local $C^{\infty}$ diffeomorphism. Thus, there exists a $C^{\infty}$ map-germ $\widetilde{\varphi}_{1}:(I, 0) \rightarrow \mathbb{R}$ such that

$$
\left.\alpha_{\mathbf{n}(0)} \circ \mathbf{n} \circ h(s)=\left(s+\widetilde{\varphi}_{1}(s), s^{2}\right)\right) \quad\left(\widetilde{\varphi}_{1}(s)=o(s)\right) .
$$

Let $\alpha_{P(0)}: H(P(0))-E_{P(0)} \rightarrow \mathbb{R} \times\{1\} \times \mathbb{R}$ be the central projection relative to $P(0)$. By the form mentioned above and Lemma 2.4, the germ of composition $\alpha_{P(0)} \circ$ ped $_{\mathbf{r}, P(0)}$ is $\mathcal{A}$-equivalent to a map-germ of the following form:

$$
s \mapsto\left(\left(s+\widetilde{\varphi}_{1}(s)\right) s^{2}, s^{2}\right) .
$$

Next, we investigate the influence of moving the pedal points $P(\lambda)$. Suppose that $P(0)=\mathbf{r}(0)$ and $P$ has $(k+1)$-point contact with $\mathbf{r}$ at $0 \in J(k \geq 0)$. In other words, suppose that there exist a sufficiently small neighborhood $U$ of $\mathbf{r}(0)$ in $S^{2}$ and a $C^{\infty}$ function $F: U \rightarrow \mathbb{R}$ such that $F \circ \mathbf{r}(s) \equiv 0\left(\forall s \in I \cap \mathbf{r}^{-1}(U)\right), F \circ P(0)=(F \circ P)^{\prime}(0)=\cdots=(F \circ P)^{(k)}(0)=0$, and $(F \circ P)^{(k+1)}(0) \neq 0$. Since $\mathbf{r}: I \rightarrow S^{2}$ is a unit speed curve, it can be assumed that $F$ is non-singular provided that $I$ (resp., $U$ ) is a sufficiently small neighborhood of 0 (resp., $\mathbf{r}(0))$. Then, there exists a sufficiently small neighborhood $\widetilde{U} \subset U$ of $\mathbf{r}(0)$ such that for any $X \in \widetilde{U}$, the integral curve of $-\operatorname{grad}(F)$ starting from $X$ lies within $\widetilde{U}$ until it reaches the image of the unit speed curve $\mathbf{r}(I)$. Let this reaching point be denoted by $\gamma(X)$ and define the map $\Gamma: \widetilde{U} \rightarrow I$ as $\Gamma(X)=\mathbf{r}^{-1} \circ \gamma(X)$. Then, $(\widetilde{U},(\Gamma, F))$ can be used as a chart at $\mathbf{r}(0)$ since the map $(\Gamma, F): \widetilde{U} \rightarrow I \times \mathbb{R}$ is non-singular. By using the chart $(\widetilde{U},(\Gamma, F))$ and by the proof of assertion 5 of Theorem 1 , the germ of composition

$$
(s, \lambda) \mapsto\left(\alpha_{P(0)} \circ \operatorname{ped}_{\mathbf{r}, P(\lambda)} \circ h(s), \lambda\right)
$$

is $\mathcal{A}$-equivalent to a map-germ of the following form:

$$
(s, \lambda) \mapsto\left(\left(s+\widetilde{\varphi}_{1}(s)\right)\left(s^{2} \pm F \circ P(\lambda)\right), s^{2} \pm F \circ P(\lambda), \lambda\right) .
$$

Furthermore, by the Malgrange preparation theorem and Theorem 3, a map-germ of the form (a) must be $\mathcal{A}$-equivalent to the map-germ $f_{k}^{ \pm}(s, \lambda)=\left(s\left(s^{2} \pm \lambda^{k+1}\right), s^{2}, \lambda\right)$.

Conversely, we suppose that the germ of pedal unfolding $U n$-ped $d_{\mathbf{r}, P}:(I \times J,(0,0)) \rightarrow S^{2} \times J$ is $\mathcal{A}$-equivalent to $S_{k}^{ \pm}(k \geq 0), P(0)=\mathbf{r}(0)$ and that $P$ does not have $(k+1)$-point contact with $\mathbf{r}$ at $0 \in J$. Then, by the proof presented above, for any positive integer $\ell, P$ does not have $(\ell+1)$-point contact with $\mathbf{r}$ at $0 \in J$. In particular, there exists a $C^{\infty}$ immersion $\widetilde{P}: J \rightarrow S^{2}$ such that $\widetilde{P}$ is sufficiently near $P$ under the Whitney $C^{\infty}$ topology, and $\widetilde{P}$ has $(k+2)$-point contact with $\mathbf{r}$ at $0 \in J$. By the proof of the implication described above, it can be concluded that $S_{k}^{ \pm}$singularity is adjacent to $S_{k+1}^{ \pm}$singularity; however, this contradicts the adjacency diagram given in [15].

Proof of assertion 4 of Theorem 1.

Suppose that the map-germ $U n$-ped $d_{\mathbf{r}, P}:(I \times J,(0,0)) \rightarrow S^{2} \times J$ is $\mathcal{A}$-equivalent to one of $B_{k}^{ \pm}, C_{k}^{ \pm}, F_{4}$, and $H_{k}$. Then, by assertions 1,2 , and 3 of Theorem 1 , the given immersion $P: J \rightarrow S^{2}$ must satisfy not only $P(0)=\mathbf{r}(0)$ but also the condition that for any positive integer $\ell, P$ does not have $(\ell+1)$-point contact with $\mathbf{r}$ at $0 \in J$. Thus, for any positive integer $\ell$, there exists a $C^{\infty}$ immersion $\widetilde{P}: J \rightarrow S^{2}$ such that $\widetilde{P}$ is sufficiently near $P$ under the Whitney $C^{\infty}$ topology, and $\widetilde{P}$ has the $(\ell+1)$-contact with $\mathbf{r}$ at $0 \in J$. Hence, it can be concluded that one of $B_{k}^{ \pm}, C_{k}^{ \pm}, F_{4}$, and $H_{k}$ singularity is adjacent to $S_{\ell}^{ \pm}$singularity for any positive integer $\ell$; however, this contradicts the adjacency diagram given in [15]. 


\section{Proof of Theorem 2}

Since $\mathbf{r}(0)$ is an ordinary inflection point, by Lemma 2.2 and the Malgrange preparation theorem, the dual germ $\mathbf{n}:(I, 0) \rightarrow S^{2}$ is $\mathcal{A}$-equivalent to the ordinary cusp $s \mapsto\left(s^{3}, s^{2}\right)$.

Proof of assertion 1 of Theorem 2.

Suppose that $P(0)$ does not belong to $E_{\mathbf{n}(0)}$. Then, for any sufficiently small $\lambda_{0} \in J, P\left(\lambda_{0}\right)$ lies outside $E_{\mathbf{n}(0)}$. This implies that by Lemma 2.4, the map-germ $\Psi_{P\left(\lambda_{0}\right)}$ at $\mathbf{n}(0)$ is non-singular. Thus, by Lemma 2.3, the map-germ $\operatorname{ped}_{\mathbf{r}, P\left(\lambda_{0}\right)}:(I, 0) \rightarrow S^{2}$ is also $\mathcal{A}$-equivalent to the ordinary cusp. Therefore, by Theorem 3, the map-germ $U n$-ped $d_{\mathbf{r}, P}:(I \times J,(0,0)) \rightarrow S^{2} \times J$ is $\mathcal{A}$-equivalent to the cuspidal edge.

Conversely, suppose that the map-germ $U n$-ped $d_{\mathbf{r}, P}:(I \times J,(0,0)) \rightarrow S^{2} \times J$ is $\mathcal{A}$-equivalent to the map-germ $g(s, \lambda)=\left(s^{3}, s^{2}, \lambda\right)$; we show that $P(0) \in E_{\mathbf{n}(0)}$ implies a contradiction under this assumption. The property $P(0) \in E_{\mathbf{n}(0)}$ implies that $\mathbf{n}(0) \in E_{P(0)}$. Since the dual germ $\mathbf{n}:(I, 0) \rightarrow S^{2}$ is $\mathcal{A}$-equivalent to the ordinary cusp $s \mapsto\left(s^{3}, s^{2}\right)$, by Lemma $2.4, \mathbf{n}(0) \in E_{P(0)}$ implies that $j^{3}\left(U n\right.$-ped $\left.d_{\mathbf{r}, P}\right)(0)$ is not $\mathcal{A}^{3}$-equivalent to $j^{3} g(0)$. This contradicts the assumption that $U n$-ped $d_{\mathbf{r}, P}:(I \times J,(0,0)) \rightarrow S^{2} \times J$ is $\mathcal{A}$-equivalent to the map-germ $g(s, \lambda)=\left(s^{3}, s^{2}, \lambda\right)$.

Proof of "if" parts of assertions 2 and 3 of Theorem 1.

Since $P(0)$ belongs to $E_{\mathbf{n}(0)}-\{\mathbf{r}(0)\}$, by composing an appropriate rotation without the loss of generality, it can be assumed that $\mathbf{n}(0)=(-1,0,0)$ and $P(0)=(0,0,1)$. Let $\alpha_{\mathbf{n}(0)}$ : $H(\mathbf{n}(0))-E_{\mathbf{n}(0)} \rightarrow\{-1\} \times \mathbb{R}^{2}$ be the central projection relative to $\mathbf{n}(0)$. Then, by Lemma 2.2, the germ of composition $\alpha_{\mathbf{n}(0)} \circ \mathbf{n}$ is of the form

$$
\alpha_{\mathbf{n}(0)} \circ \mathbf{n}(s)=\left(a s^{2}+b s^{3}+\varphi_{1}(s), c s^{2}+d s^{3}+\varphi_{2}(s)\right),
$$

where $b c \neq 0$ and $\varphi_{j}(s)=o\left(s^{3}\right)$. Since $c \neq 0$, there exists a germ of $C^{\infty}$ diffeomorphism $h:(I, 0) \rightarrow(I, 0)$ such that

$$
\alpha_{\mathbf{n}(0)} \circ \mathbf{n} \circ h(s)=\left(\widetilde{a} s^{2}+\widetilde{b} s^{3}+\widetilde{\varphi}_{1}(s), s^{2}\right),
$$

where $\widetilde{b} \neq 0$ and $\widetilde{\varphi}_{1}(s)=o\left(s^{3}\right)$. Let $\alpha_{P(0)}: H(P(0))-E_{P(0)} \rightarrow \mathbb{R}^{2} \times\{1\}$ be the central projection relative to $P(0)$. By the form mentioned above and Lemma 2.4, the germ of composition $\alpha_{P(0)} \circ$ $\operatorname{ped}_{\mathbf{r}, P(0)}$ is $\mathcal{A}$-equivalent to a map-germ of the following form:

$$
s \mapsto\left(\left(\widetilde{a} s^{2}+\widetilde{b} s^{3}+\widetilde{\varphi}_{1}(s)\right) s^{2}, s^{2}\right) .
$$

Next, we investigate the influence of moving the pedal points $P(\lambda)$. Suppose that $P(0)=\mathbf{r}(0)$ and $P$ has $(k+1)$-point contact with $E_{\mathbf{n}(0)}$ at $0 \in J(k \geq 0)$. Since $E_{\mathbf{n}(0)}$ is defined by the equation $\mathbf{n}(0) \cdot X=0$, the assumption of $(k+1)$-point contact implies that $\mathbf{n}(0) \cdot P(0)=\mathbf{n}(0) \cdot P^{\prime}(0)=$ $\cdots=\mathbf{n}(0) \cdot P^{(k)}(0)=0$ and $\mathbf{n}(0) \cdot P^{(k+1)}(0) \neq 0$. Then, as in the proof of assertions 2 and 3 of Theorem 1, the germ of composition

$$
(s, \lambda) \mapsto\left(\alpha_{P(0)} \circ \operatorname{ped}_{\mathbf{r}, P(\lambda)}(s), \lambda\right)
$$

is $\mathcal{A}$-equivalent to the germ of the following form:

$$
(s, \lambda) \mapsto\left(\left(\widetilde{a} s^{2}+\widetilde{b} s^{3}+\varphi_{1}(s)\right)\left(s^{2} \pm \mathbf{n}(0) \cdot P(\lambda)\right), s^{2} \pm \mathbf{n}(0) \cdot P(\lambda), \lambda\right) .
$$

Furthermore, by the Malgrange preparation theorem and Theorem 3, a map-germ of the form mentioned in (b) must be $\mathcal{A}$-equivalent to the map-germ $g_{k}^{ \pm}(s, \lambda)=\left(s^{3}\left(s^{2} \pm \lambda^{k+1}\right), s^{2}, \lambda\right)$. 
The "only if" parts of assertions 2 and 3 of Theorem 2 can be proved as follows. Put $\widetilde{g}(s, \lambda)=$ $s^{2}, \widetilde{g}_{0}(s, \lambda)=s^{4}+\lambda s^{2}, \widetilde{g}_{2 i+1}^{ \pm}(s, \lambda)=s^{4} \pm \lambda^{2 i+2} s^{2}$, and $\widetilde{g}_{2 i}^{+}(s, \lambda)=s^{4}+\lambda^{2 i+1} s^{2}$. Then, it can be clearly seen that any two distinct elements of the following set are not $\mathcal{K}^{T}$-equivalent.

$$
\left\{\widetilde{g}, \widetilde{g}_{0}, \widetilde{g}_{1}^{+}, \widetilde{g}_{1}^{-}, \widetilde{g}_{2}^{+}, \widetilde{g}_{3}^{+}, \widetilde{g}_{3}^{-}, \cdots\right\} .
$$

Hence, by Theorem 3, any two distinct elements of the set of the cuspidal edge, cuspidal crosscap, cuspidal $S_{1}^{+}$, cuspidal $S_{1}^{-}$, cuspidal $S_{2}^{+}$, cuspidal $S_{3}^{+}$, cuspidal $S_{3}^{-} \cdots$ are not $\mathcal{A}$-equivalent. Furthermore, by Theorem 3 and the form of $g_{0}, g_{1}^{ \pm}, g_{2}^{ \pm}, \ldots$ in Table 2, the following adjacency diagram is obtained.

$$
\cdots \longrightarrow \text { cuspidal } S_{k} \longrightarrow \cdots \longrightarrow \text { cuspidal } S_{1} \longrightarrow \text { cuspidal } S_{0} .
$$

Proof of "only if" parts of the assertions 2, 3 of Theorem 2.

As in the proof of the "only if" parts of assertions 2 and 3 of Theorem 1, we suppose that $U n$ $\operatorname{ped}_{\mathbf{r}, P}:(I \times J,(0,0)) \rightarrow S^{2} \times J$ is $\mathcal{A}$-equivalent to cuspidal $S_{k}^{ \pm}(k \geq 0), P(0) \in E_{\mathbf{n}(0)}-\{\mathbf{r}(0)\}$, and $P$ does not have $(k+1)$-point contact with $E_{\mathbf{n}(0)}$ at $0 \in J$. Then, by the "if" parts of assertions 2, 3 of Theorem 2, for any non-negative integer $\ell, P$ does not have $(\ell+1)$-point contact with $E_{\mathbf{n}(0)}$ at $0 \in J$. In particular, for any non-negative integer $\ell$, there exists a $C^{\infty}$ immersion $\widetilde{P}: J \rightarrow S^{2}$ such that $\widetilde{P}$ is sufficiently near $P$ under the Whitney $C^{\infty}$ topology, and $\widetilde{P}$ has $(\ell+1)$-point contact with $E_{\mathbf{n}(0)}$ at $0 \in J$. Hence, it can be concluded that cuspidal $S_{k}^{ \pm}$singularity is adjacent to cuspidal $S_{\ell}^{ \pm}$singularity for any positive integer $\ell$; however, this contradicts diagram (c).

Next, suppose that $U n$-ped $d_{\mathbf{r}, P}:(I \times J,(0,0)) \rightarrow S^{2} \times J$ is $\mathcal{A}$-equivalent to cuspidal $S_{k}^{ \pm}$ $(k \geq 0)$ and $P(0)=\{\mathbf{r}(0)\}$. In this case, the tangent cone of $\mathbf{n}(I)$ at $\mathbf{n}(0)$ coincides with $E_{P}$. Thus, by Lemma $2.4, j^{2}\left(U n-p e d_{\mathbf{r}, P}\right)(0)$ is not $\mathcal{A}^{2}$-equivalent to $j^{2} g_{k}^{ \pm}(0)$; this contradicts the assumption that $U n$-ped $d_{\mathbf{r}, P}:(I \times J,(0,0)) \rightarrow S^{2} \times J$ is $\mathcal{A}$-equivalent to the map-germ $g_{k}^{ \pm}(s, \lambda)=\left(s^{5} \pm \lambda^{k+1} s^{3}, s^{2}, \lambda\right)$.

\section{Remarks}

It is possible to adopt the criteria given in [16] or an argument similar to that given in [7] to prove Theorems 1 and 2. However, the criteria in [16] are too general to be directly applied to our study, and the argument in [7] seems to be somewhat ad hoc. Thus, in order to apply them to our study, considerable preliminary work is required, the proofs of which are time-consuming and complicated. On the other hand, Theorem 3 is the most suitable criterion for our study. Moreover, the calculations with respect to $\mathcal{K}^{T}$-equivalence are relatively straightforward; hence, by using Theorem 3, we can prove both Theorem 1 and Theorem 2 in a coherent and unified manner.

\section{REFERENCES}

[1] V. I. Arnol'd, The geometry of spherical curves and the algebra of quaternions, Russian Math. Surveys, 50 (1995), 1-68. DOI: 10.1070/RM1995v050n01ABEH001662

[2] V. I. Arnol'd, Singularities of Caustics and Wave fronts, Springer, Berlin-New York, 2001.

[3] V. I. Arnol'd, V. V. Goryunov, O. V. Lyashko, V. A. Vasil'ev, Dynamical Systems VIII, Encyclopaedia of Mathematical Sciences, 39, Springer-Verlag, Berlin Heidelberg New York, 1989.

[4] V. I. Arnol'd, S. M. Gusein-Zade, A. N. Varchenko, Singularities of Differentiable Maps I, Monographs in Mathematics, 82, Birkhäuser, Boston Basel Stuttgart, 1985.

[5] J. W. Bruce, P. J. Giblin, Curves and Singularities (second edition), Cambridge University Press, Cambridge, 1992.

[6] X. Y. Chen and T. Matumoto, On generic 1-parameter families of $C^{\infty}$-maps of an n-manifold into a $(2 n-1)$-manifold, Hiroshima Math. J., 14 (1984), 547-550. 
[7] S. Fujimori, K. Saji, M. Umehara and K. Yamada, Cuspidal cross caps and singularities of maximal surfaces, Math. Z., 259 (2008), 827-848. DOI: 10.1007/s00209-007-0250-0

[8] T. Gaffney, On the order of determination of a finitely determined germs, Invent. Math., 37 (1976), 83-92. DOI: $10.1007 / \mathrm{BF} 01418963$

[9] T. Gaffney, A note on the order of determination of a finitely determined germs, Invent. Math., 59 (1979), 127-130. DOI: 10.1007/BF01403059

[10] T. Gaffney and A. A. du Plessis, More on the determinacy of smooth map-germs, Invent. Math., 66 (1982), 137-163. DOI: 10.1007/BF01404761

[11] S. Izumiya, K. Saji and M. Takahashi, Horospherical flat surfaces in hyperbolic 3-space, J. Math. Soc. Japan, 62 (2010), 789-849. DOI: 10.2969/jmsj/06230789

[12] S. Izumiya and F. Tari, Projections of hypersurfaces in the hyperbolic space to hyperhorospheres and hyperplanes, Rev. Mat. Iberoam. 24 (2008) 895-920.

[13] J. Mather, Stability of $C^{\infty}$ mappings, III, Finitely determined map-germs, Publ. Math. I. H. E. S., 35 (1969), 127-156. DOI: 10.1007/BF02698926

[14] J. Mather, Stability of $C^{\infty}$ mappings, IV, Classification of stable map-germs by $\mathbf{R}$-algebras, Publ. Math. I. H. E. S., 37 (1970), 223-248. DOI: 10.1007/BF02684889

[15] D. Mond, On the classification of germs of maps from $\mathbb{R}^{2}$ to $\mathbb{R}^{3}$, Proc. London Math. Soc., 50 (1985), 333-369. DOI: $10.1112 / \mathrm{plms} / \mathrm{s} 3-50.2 .333$

[16] T. Nishimura, Criteria for right-left equivalence of smooth map-germs, Topology, 40 (2001), $433-462$. DOI: 10.1016/S0040-9383\%2899\%2900068-3

[17] T. Nishimura, Normal forms for singularities of pedal curves produced by non-singular dual curve germs in $S^{n}$, Geom Dedicata, 133 (2008), 59-66. DOI: 10.1007/s10711-008-9233-5

[18] T. Nishimura, Singularities of pedal curves produced by singular dual curve germs in $S^{n}$, Demonstratio Math., 43 (2010), 447-459.

[19] T. Nishimura and K. Kitagawa, Classification of singularities of pedal curves in $S^{2}$, The Natural Sciences, Journal of the Faculty of Education and Human Sciences, Yokohama National University, 10 (2008), 39-55. (available from http://hdl.handle.net/10131/4067).

[20] A. A. du Plessis, On the determinacy of smooth map-germs, Invent. Math., 58 (1980), 107-160. DOI: $10.1007 / \mathrm{BF} 01403166$

[21] I. R. Porteous, Geometric Differentiation (second edition), Cambridge University Press, Cambridge, 2001.

[22] K. Saji, Criteria for cuspidal $S_{k}$ singularities and its applications, preprint.

[23] C. T. C. Wall, Finite determinacy of smooth map-germs, Bull. London Math. Soc., 13 (1981), 481-539. DOI: $10.1112 / \mathrm{blms} / 13.6 .481$

Department of Mathematics,

Faculty of Education and Human Sciences,

Yokohama National University, Yokohama240-8501, Japan e-mail: takashi@edhs.ynu.ac.jp 